OPEN

SUBJECT AREAS:

COMPUTATIONAL

METHODS

BIOSURFACES

Received

14 October 2013

Accepted

15 January 2014

Published

3 February 2014

Correspondence and requests for materials should be addressed to H.L. (lihuilmy@hotmail.

com)

\title{
Dewetting Properties of Metallic Liquid Film on Nanopillared Graphene
}

\author{
Xiongying Li, Yezeng He, Yong Wang, Jichen Dong \& Hui Li
}

Key Laboratory for Liquid-Solid Structural Evolution and Processing of Materials, Ministry of Education, Shandong University, Jinan 250061 , P. R. China.

In this work, we report simulation evidence that the graphene surface decorated by carbon nanotube pillars shows strong dewettability, which can give it great advantages in dewetting and detaching metallic nanodroplets on the surfaces. Molecular dynamics (MD) simulations show that the ultrathin liquid film first contracts then detaches from the graphene on a time scale of several nanoseconds, as a result of the inertial effect. The detaching velocity is in the order of $10 \mathrm{~m} / \mathrm{s}$ for the droplet with radii smaller than $50 \mathrm{~nm}$.

Moreover, the contracting and detaching behaviors of the liquid film can be effectively controlled by tuning the geometric parameters of the liquid film or pillar. In addition, the temperature effects on the dewetting and detaching of the metallic liquid film are also discussed. Our results show that one can exploit and effectively control the dewetting properties of metallic nanodroplets by decorating the surfaces with nanotube pillars.

W ettability is a fundamental property of a solid surface, and plays important roles in daily life, industry and agriculture. The amazing climbing ability of geckos ${ }^{1-3}$, as well as the self-cleaning effects of lotus leaves and butterfly wings ${ }^{4,5}$, are all related to the unique micro- and nanostructures on their surfaces. Inspired by these strategies, functional surfaces with special wettability, such as nanopillared surfaces, have elicited great research interest ${ }^{6-9}$ and been synthesized by various methods ${ }^{10-14}$. For example, the superhydrophilic surface ${ }^{15,16}$ with a water contact angle of almost $0^{\circ}$ fabricated by UV irradiation has been successfully used as a transparent coating with antifogging and self-cleaning properties. On the other hand, various phenomena, such as contamination, snow sticking, erosion and water transport are expected to be inhibited on superhydrophobic surfaces ${ }^{17-19}$ with a contact angle larger than $150^{\circ}$.

Much research has been performed to investigate the wetting behavior of liquid nanostructures, including water, polymers and metals ${ }^{20-28}$. In terms of metallic thin films, these materials have attracted much attention not only due to their central importance for thin film technology, such as controlling the homogeneity of thin films and coatings $\mathrm{s}^{29,30}$, but also because of their potential applications in catalysis, photovoltaics, sensor, transparent conductors and memory cells ${ }^{31-33}$. Due to the dramatic difference in the physical properties relative to typical polymer and water films, including high surface energy, low viscosity, and differences in the long range interaction forces, continuous metallic films contract far from equilibrium, and are unstable with respect to forming discrete nanodroplets via dewetting ${ }^{34}$. Numerous experimental and theoretical investigations have confirmed that the dewetting begins at the edges or corners of films where the surface tension is high $^{35-37}$, and the dewetting evolution undergoes three successive stages: rupture of the film resulting in the occurrence of nanoholes, growth of the holes followed by the mass and energy transmissions, and finally the decay of the holes. According to hydromechanics, inertia plays a dominant role in the dewetting dynamics of metallic nanostructures ${ }^{36,37}$. Recently, a unique property of these films, i.e. jumping off the substrates, has been reported. Habenicht et al. ${ }^{36}$ first demonstrated that deformed Au droplets could convert their surface deformation energy into kinetic energy, which makes them contract and finally detach from the non-wettable substrate. Fuentes-Cabrera et al. ${ }^{38}$, Afkhami and $\mathrm{Kondic}^{37}$ have reported that the evolution of the liquid $\mathrm{Cu}$ film is strongly dependent on its initial shape and the contact angle (CA). Such a jumping phenomenon had also been found in the evaporation of water droplets on $\mathrm{G}^{39}$. These important findings shed new light on the interfacial interactions and possibly pave the way for selfassembly nanotechnology ${ }^{40-42}$.

Much research has been done for the interfacial behavior of graphene, such as wetting and applications of super-hydrophobicity in self-cleaning materials, however, to our knowledge, fewer efforts have focused on the dewetting-induced detachment of nanodroplets, especially how to use super-hydrophobicity to control the movement of nanodroplets. Wetting and dewetting are important practical issues in physical and material sciences, and provide an opportunity for comprehensive and satisfactory understanding of the interfacial 
(a)

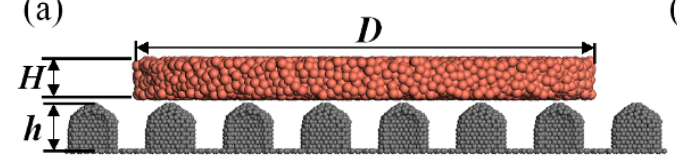

(b)

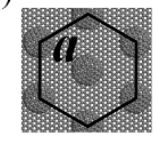

Figure $1 \mid$ Schematic plot of the simulated system. The capped carbon nanotubes (CNTs) are placed at the corners and center of the regular hexagons on the graphene to form a nanopillared graphene (PG), mimicking the super dewetting surfaces. The interval distance, diameter and hight of pillars are defined as $a, d$ and $h$, respectively. The diameter and thickness of the disk are denoted as $D$ and $H$, respectively.

properties of nanomaterials. As of yet, although there has been much progress in the understanding of dewetting, some important questions concerning the super-hydrophobic surfaces remain unanswered. Motivated by this and inspired by the amazing wettability of the nanofabricated surfaces mentioned above, we performed molecular dynamics (MD) simulations to explore the dewetting behavior of metallic thin films on pillared graphene (PG) consisting of uniform-distributed canbon nanotube (CNT) pillars, with the aim of controlling the contraction and detachment of liquid films by tuning the sizes of nanopillars. Figure 1 shows the model of the simulated system that a liquid $\mathrm{Cu}$ disk is deposited on a nanopillared graphene (PG) constructed with vertically aligned carbon nanotube (CNT) pillars.

\section{Results}

To determine the role of the capped CNT nanopillars on the dewetting properties of surfaces, a comparison between $G$ and PG surfaces is made. Figure 2 shows snapshots of the dewetting dynamics and potential energy distributions of liquid Cu disks with $D=135.6 \AA$. Notably, when $H=3 \AA$, the film on either the G or PG first ruptures to form some nanoholes (Figure $2 \mathrm{a}$ and $\mathrm{b}$ ). These nanoholes gradually combine into a large hole, then decay and disappear. This phenomenon provides insight into the evolution process of nanoholes within the ultrathin liquid film (see the video 1 in the Supporting Information for details of the Cu-PG system), which is in good agreement with the experimental results ${ }^{35}$. When $H$ increases to $5 \AA$, no holes can be observed. This indicates that whether the nanohole exists during the contraction is mainly dependent on the $H$ of the film rather than the surface structure. Interestingly, further research presents that the PG can detach the nanodroplet from it (see the video 2 in the Supporting Information), while this detachment can not be observed on $\mathrm{G}$, regardless of the fact that $H$ increases to $10 \AA$. Like PG, another substrate PG2 displays similar dewetting properties, as shown in Figure 2c. The droplets detach from the PG and PG2 surfaces at 41 and 51 ps, respectively. Importantly, the film sizes (initial diameter $D$, thickness $H$ and radius of droplet $R$ ) used in this study and the detaching time $t_{d}=41 \mathrm{ps}$, are far smaller than those in previous studies ${ }^{36-38,49}$. The comparison indicates that the nanopillars would improve the dewetting property of flat surfaces which brings great advantages in detaching droplets from the substrate. These findings are critical in the chemical system, daily life and nanotechnology, as well as guiding nanoparticles toward a tumor. In addition, the switch of dewetting modes with respect to the $H$ also gives us the inspiration to avoid the formation of nanoholes within the superthin films by decreasing the film sizes, which is very important in the practical applications of superthin liquid films, such as homogeneous thin film, coating ${ }^{29,30}$, anti-adsorption and self-cleaning materials.

Potential energy distributions indicate that the dewetting begins at the edges of films and nanoholes. As the simulation proceeds, the edge propagates and contracts toward the center of the disk, which makes mass and energy flow and finally forms a spherical droplet when the system approaches the equilibrium $(t=200 \mathrm{ps})$. A better understanding of the contraction and detachment of the liquid film can be gained by the energy profiles. The total potential energy, $E_{t o t}$, is defined as $E_{\text {tot }}=E_{C-C}+E_{C u-C u}+E_{C-C u}$, where $E_{C-C}, E_{C u-C u}$ and $E_{C-C u}$ represent the C-C, $\mathrm{Cu}-\mathrm{Cu}$ and $\mathrm{C}-\mathrm{Cu}$ interaction, respectively. Naturally, the $E_{C-C}$ is equal to $0 \mathrm{eV}$ due to the fix of the substrate. The $E_{C-C u}$ is a resisting force which hinders the slipping and ejection of droplets due to its reverse direction to the slipping and detachment. Therefore, $E_{\mathrm{Cu} \text {-Cu}}$ is the driving force. The energy difference, $\Delta E_{\mathrm{Cu} \text {-Cu}}$, is partially transformed into kinetic energy of the droplet, which induces contraction and even detachment. Figure 3 gives the $\Delta E_{C u-C u}, E_{C-C u}$ and $v_{z}(t)$ as a function of time. As for those systems without detachment, the $\Delta E_{\mathrm{Cu}-\mathrm{Cu}}\left(E_{\mathrm{C}-\mathrm{Cu}}\right)$ of the $\mathrm{Cu}-\mathrm{PG}$ system is much larger (lower) than the $\mathrm{Cu}-\mathrm{G}$ system, indicating that the PG exhibits a stronger dewettability than the flat $G$, as shown in Figures 3 $\mathrm{a}$ and $\mathrm{b}$. At the first $15 \mathrm{ps}$, the rapid decrease of $\Delta E_{\mathrm{Cu}-\mathrm{Cu}}$ and $E_{\mathrm{C}-\mathrm{Cu}}$ accelerates the contraction of the liquid film and helps the droplet move upward, thus leading to the rapid nearly-linear increase of $v_{z}$. It is worth noting that there exists a small peak when $H=3 \AA$

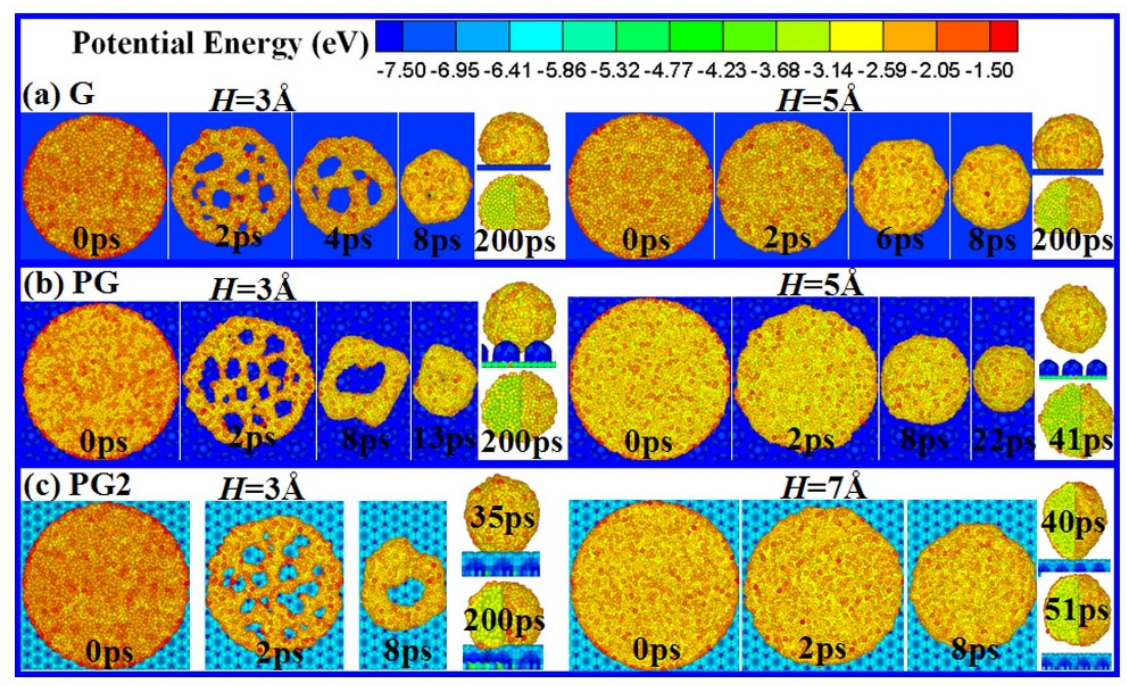

Figure $2 \mid$ Dewetting dynamics and potential energy distributions of Cu films ( $D=135.6 \AA$ ) on substrates. (a) G. (b) PG, $a=10.2$ and $h=13.67 \AA$. (c) Another pillared graphene (PG2) with the concave-cap CNT as the pillar, $a=3.4$ and $h=10.92 \AA$. For clear observation of the internal energy distribution, one fouth of some liquid droplets has been cut off. When $H$ increases to $5 \AA$, the droplet detaches from the PG surface at 41 ps due to dewetting. Further increasing $H$ to $7 \AA$, detachment occurs in the Cu-PG2 system at 51 ps. 


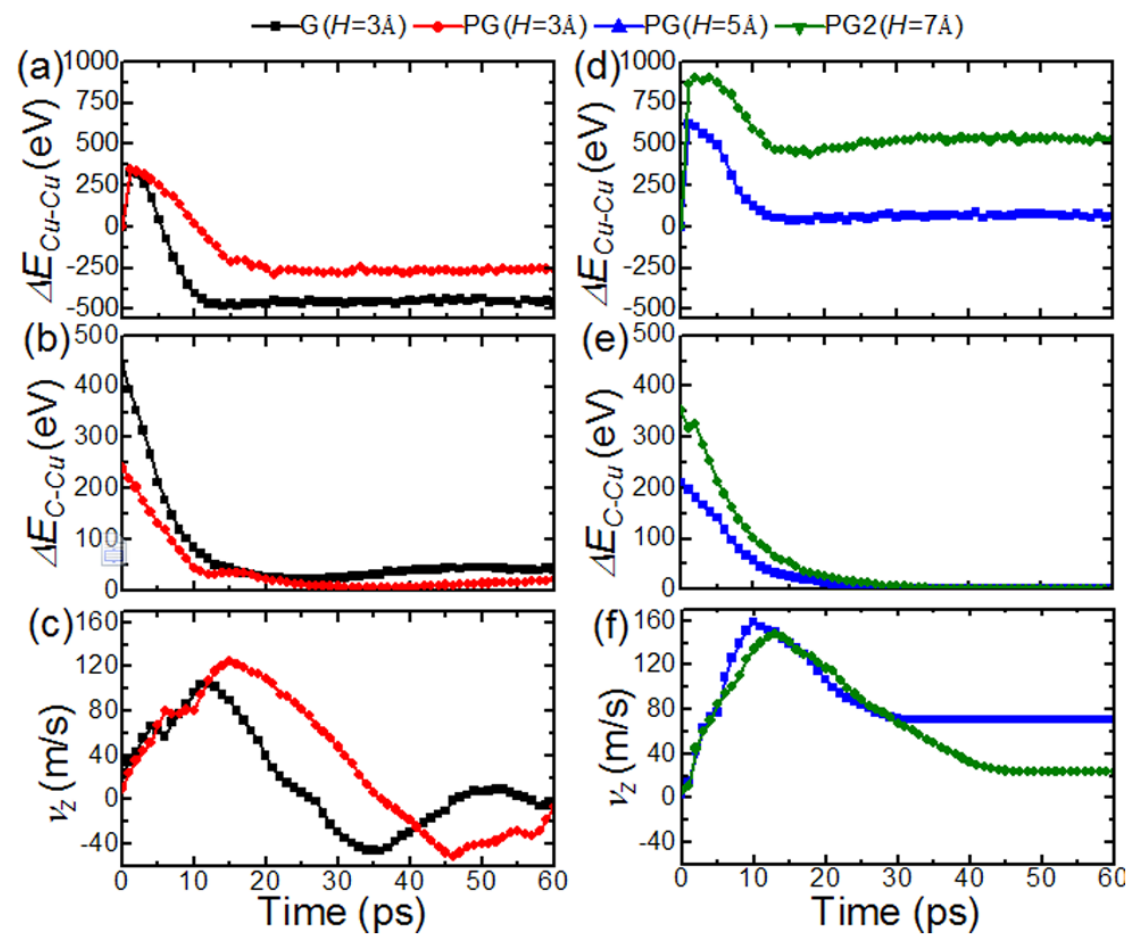

Figure $3 \mid \Delta E_{C u-C u}, E_{C-C u}$ and $v_{z}$ of the systems without (left) and with (right) detachment.

(Figure 3c), which is considered to be directly related to the existence of nanoholes, and this plays a critical role in determining the dewetting mode of metallic films. When $t=10 \sim 15 \mathrm{ps}$, the $v_{z}$ in all systems reaches the maximum. Furthermore, the peaks in the $\mathrm{Cu}$ PG system are much higher than those in the $\mathrm{Cu}-\mathrm{G}$ system, confirming that the surface nanostructures greatly influence the contracting velocity. After $15 \mathrm{ps}$, the $v_{z}$ decreases rapidly to a value far below $0 \mathrm{~m} / \mathrm{s}$ due to the combined effects of the $E_{C-C u}$ and the $\Delta E_{C u-C u}$, which signifies that the elongated droplets spread to the surfaces again and would not jump off the surface. Comparing with those systems without detachment, the systems with detachment present remarkably different results, as indicated in Figures $3 \mathrm{~d}$-f. First, the driving force $\Delta E_{\mathrm{Cu}-\mathrm{Cu}}$ is much larger either at the beginning or at the equilibrium, which is one of the key factors that the droplet could detach from the pillared surfaces rather than from the G. Second, the hindering force $E_{C-C u}$ is much lower, which is quite benefit for the droplet to contract and detach. Finally, the much higher $v_{z}$ implies that a large maximum $v_{z}$ is essential for the subsequent detachment. Therefore, from the above analysis, we regard that low $E_{C-C u}$ and high equilibrium $\triangle E_{\mathrm{Cu}-\mathrm{Cu}}$ are the key factors for the detachment. The high $\Delta E_{\mathrm{Cu} \text {-Cu}}$ could generate a large $v_{z}$, and the low $\Delta E_{C-C u}$ ensures that the $v_{z}$ remains at a constant value above zero.

There still exists the question of what plays the dominant role in determining the contraction and detachment: the inertial force, or the viscous force. To gain insight into this question, we carry out additional analysis from the perspective of hydromechanics. The Reynolds number ( $\mathrm{Re}$ ) is a dimensionless quantity which gives a measure of the ratio of inertial forces to viscous forces, and consequently quantifies the relative importance of these two types of forces during the liquid film evolution. $\operatorname{Re}$ is given as $\operatorname{Re}=\frac{\rho v L}{\mu}$, where $\rho, v$ and $\mu$ are the density, mean velocity and dynamic viscosity of the fluid, respectively, and $L$ is a characteristic linear dimension. For liquid $\mathrm{Cu}$ in these systems, $\mathrm{Re} \approx 6.10$, indicating that the inertial force plays an important role in the contraction and detachment, which accords well with the deductions from Refs. 36 and 37.

Figure 4 provides the dependency of $H$ on the detachment. Notably, as $H$ increases from 5 to $10 \AA, t_{d}$ increases monotonously, but $v_{d}$ slightly decreases with some fluctuations. At every point of $H$, the $t_{d}\left(v_{d}\right)$ decreases (increases) significantly with the increasing $a$. These phenomena indicate that the dewetting dynamics of the $\mathrm{Cu}$ liquid film is more sensitive to the $a$ than $H$. That is because increasing every $1 \AA$ of the $H$ results in a much larger increase of the $E_{C \text {-Cu }}$ than the $E_{\mathrm{Cu}-\mathrm{Cu}}$, however, increasing a conversely leads to the decrease of the hindering force $E_{C-C u}$. That is, decreasing the $E_{C-C u}$ is the key to effectively detach the liquid droplet. Therefore, we can conclude from Figure 4 that it is an effective means to control the dewetting dynamics of the liquid film by tailing the interval distance of pillars rather than tuning the initial $H$ of the films.

The above analysis indicates the great effect of $a$ on detachment. Actually, the height $h$ of pillars may also affect the dewettability. To verify this notion, a series of simulations are carried out on the liquid evolution of $\mathrm{Cu}$ films on PGs with $h$ ranging from 13.67 to $17.22 \AA$. Different from the $a$, the $h$ presents slight dependency on the detachment. Figure $5 \mathrm{a}$ and $\mathrm{b}$ shows the influence of $a$ and $h$ on the $t_{d}$ and $v_{d}$. Clearly, the $t_{d}$ exhibits a nearly exponential decrease and $v_{d}$ shows a exponential increase with the increasing $a$. However, when $a$ is fixed, both the $t_{d}$ and $v_{d}$ change slightly with the $h$ increasing from 13.67 to $17.22 \AA$. It indicates that the detaching properties of the droplet is not sensitive to the height of the pillars, which may bring interesting insights to the design of novel microfluidic devices.
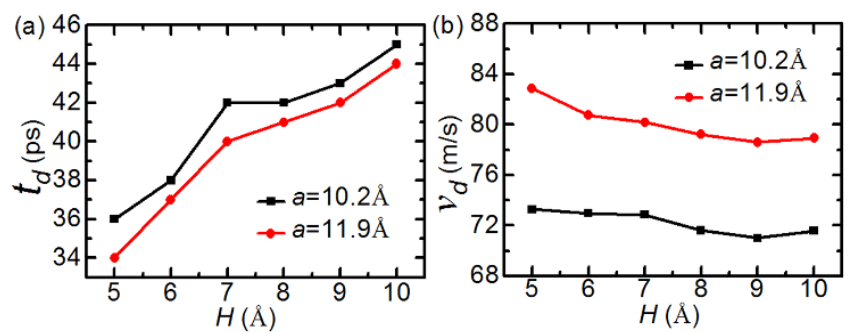

Figure $4 \mid$ The $H$ effects on the detaching behavior $t_{d}(a)$ and $v_{d}(b)$. The increasing tendency of $t_{d}$ and slightly decreasing tendency of $v_{d}$ reflect the important role of $H$ in determining the wetting dynamics. 

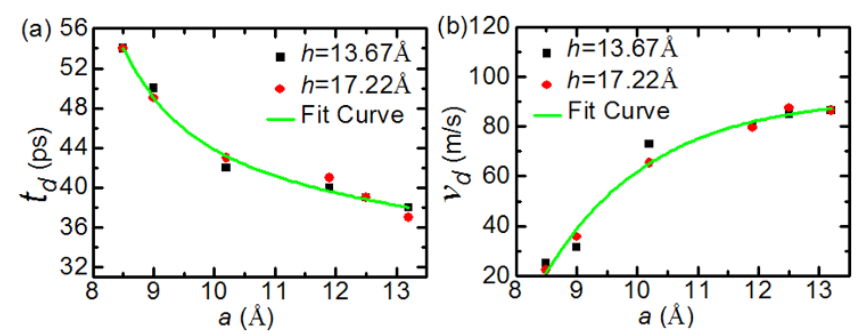

Figure $5 \mid$ The size effects of nanopillars on detachment: $t_{d}(\mathrm{a})$ and $v_{d}(\mathrm{~b})$. The solid lines are fit curves, which reveal that the $t_{d}\left(v_{d}\right)$ exponentially increases (decreases) with the interval distance of pillars $a$.

To explore how the shape of the liquid film affects the dewetting behavior, a comparison among the $\mathrm{Cu}$ films in the shapes of disk, square and triangle is made. Figure 6 shows snapshots of these films, as well as the corresponding $t_{d}$ and $v_{d}$. From the top view of the dewetting process, it can be seen that the Cu disk always remains circular in shape, and the square $\mathrm{Cu}$ forms a circle at about $23 \mathrm{ps}$, but more than 23 ps for the triangle $\mathrm{Cu}$. Interestingly, the snapshot of the triangle $\mathrm{Cu}$ clearly indicates that the contraction at the straight lines is faster than that at the corners. Importantly, the $t_{d}(62 \mathrm{ps})$ for the triangle $\mathrm{Cu}$ is largest, followed by 58 ps for the square, then by 49 ps for the disk. The comparison between these films strongly suggests that the sharp corner does not favor the contraction and detachment. That is, the dewetting behavior of liquid films shows significant dependence on their initial shapes (Figures $6 \mathrm{a}-\mathrm{c}$ ), which is further confirmed by Figures $6 \mathrm{~d}-\mathrm{f}$. Notably, after $12 \mathrm{ps}$, the $v_{z}$ for the disk $\mathrm{Cu}$ films is the largest and reaches the maximum of about $128 \mathrm{~m} / \mathrm{s}$ at $21 \mathrm{ps}$, but the $v_{z}$ for the square Cu slightly decreases then increases to a much lower maximum of about $114 \mathrm{~m} / \mathrm{s}$ at $24 \mathrm{ps}$, especially, the $v_{z}$ for the triangle $\mathrm{Cu}$ decreases greatly then slightly increases to the lowest maximum of about 93 at 28 ps (Figure 6d). Moreover, at every point of $D$, the $t_{d}\left(v_{d}\right)$ of the triangle and square $\mathrm{Cu}$ is much larger (smaller) than those of the disk $\mathrm{Cu}$ (Figures $6 \mathrm{e}$ and $\mathrm{f}$ ). These shape effects may possibly be explained by the fact that the sharp corners consume more formation energy to form a circle than the smooth lines during the dewetting process. Additionally, we also note that both $t_{d}$ and $v_{d}$ increase with the increasing $D$, suggesting that increasing $D$ would delay the detachment but increase the $v_{d}$, due to the increase of the surface energy.

To investigate the influence of temperature $(T)$ on the $t_{d}$ and $v_{d}$, we have carried out a series of MD simulations of a Cu disk with $D=$
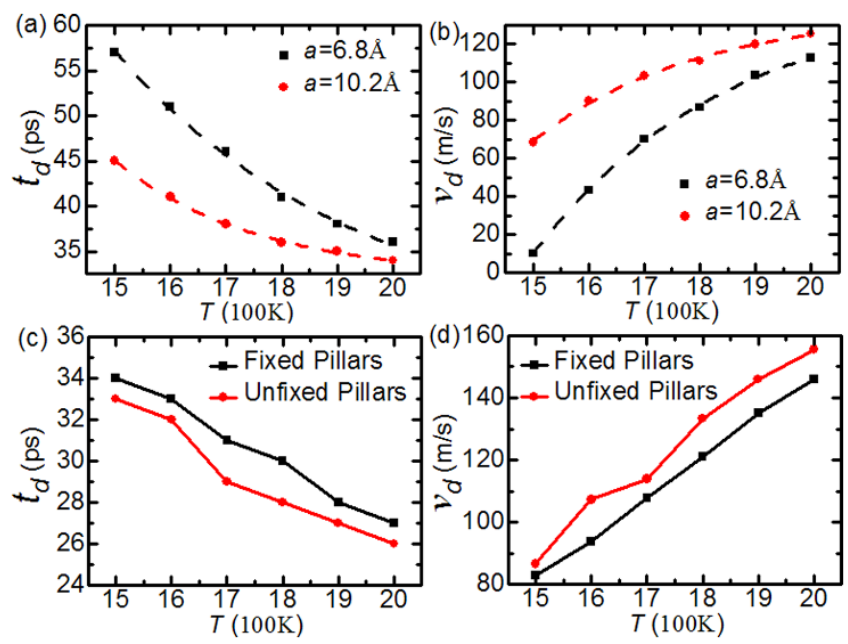

Figure $7 \mid$ The temperature effects on detachment. (a) and (b) The curves correspond to those systems with $H=10 \AA, h=13.67 \AA$, and $a$ ranging from 6.8 to $10.2 \AA$. The dashed lines are fit curves, which reveal that the $t_{d}$ $\left(v_{d}\right)$ presents an exponential relation with $T$. (c) and (d) The effect of the surface structure change on the detaching properties at high temperatures. The sample used here corresponds to the system $a=11.9 \AA, h=13.67 \AA$ and $H=5.0 \AA$. Results show that the $t_{d}$ decreases $4.36 \%$ and the $v_{d}$ increases $8.12 \%$ after considering the surface structure change, indicating that it is relatively easy for the $\mathrm{Cu}$ droplet to jump off with the consideration of the structure change.

135.6 $\AA$ on the PG at temperatures ranging from 1500 to $2000 \mathrm{~K}$. As shown in Figures $7 \mathrm{a}$ and b, increasing T from 1500 to $2000 \mathrm{~K}$ leads to a $24.44 \%$ decrement of $t_{d}$ and $82.76 \%$ increment of $v_{d}$ for $a=10.2 \AA$, indicating that the detachment can be controlled by adjusting the temperature. These temperature effects are attributed to the enhanced inertial effects, which result from the decreases of density and viscosity due to the increase of $T^{39}$. As an example, when $T$ increases from 1500 to $1900 \mathrm{~K}$, the $R e$ increases from 6.10 to 10.13 , indicating that the role of inertial effects is very remarkable at high temperatures. The effect of the surface structure change of the PG at high temperatures is presented in Figures $7 \mathrm{c}$ and $\mathrm{d}$, which show that the detaching time $\left(t_{d}\right)$ averagely decreases $4.36 \%$ and the detaching velocity $\left(v_{d}\right)$ averagely increases $8.12 \%$, indicating that it is slightly easy for the $\mathrm{Cu}$ droplet to detach from the substrate if the surface structure changes at high temperatures.

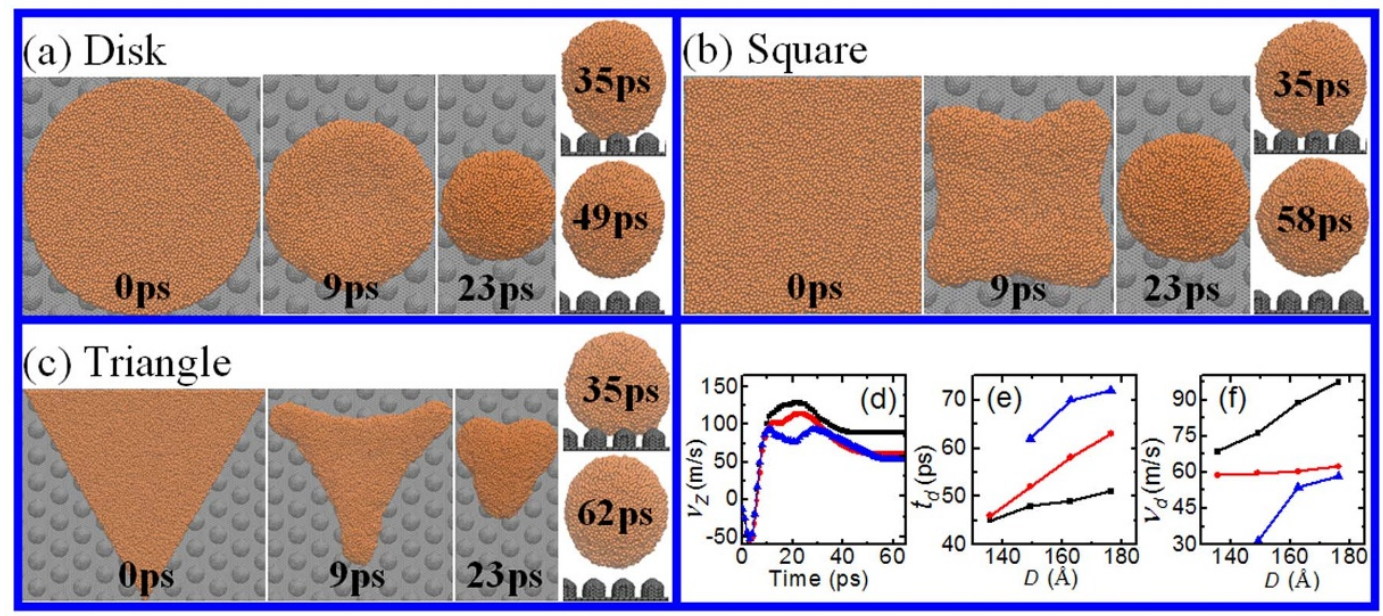

Figure $6 \mid$ The initial shape effects of $\mathrm{Cu}$ films on detachment. The $H$ is fixed at $10 \AA$. (a)-(c) Snapshots of the detaching process. (d) The $v_{z}(t)$ of the liquid Cu droplet when $D=162.72 \AA$. (e) and (f) $t_{d}$ and $v_{d}$ as functions of $D$. The black, red and blue lines stand for the results of the disk, square and triangle, respectively. 

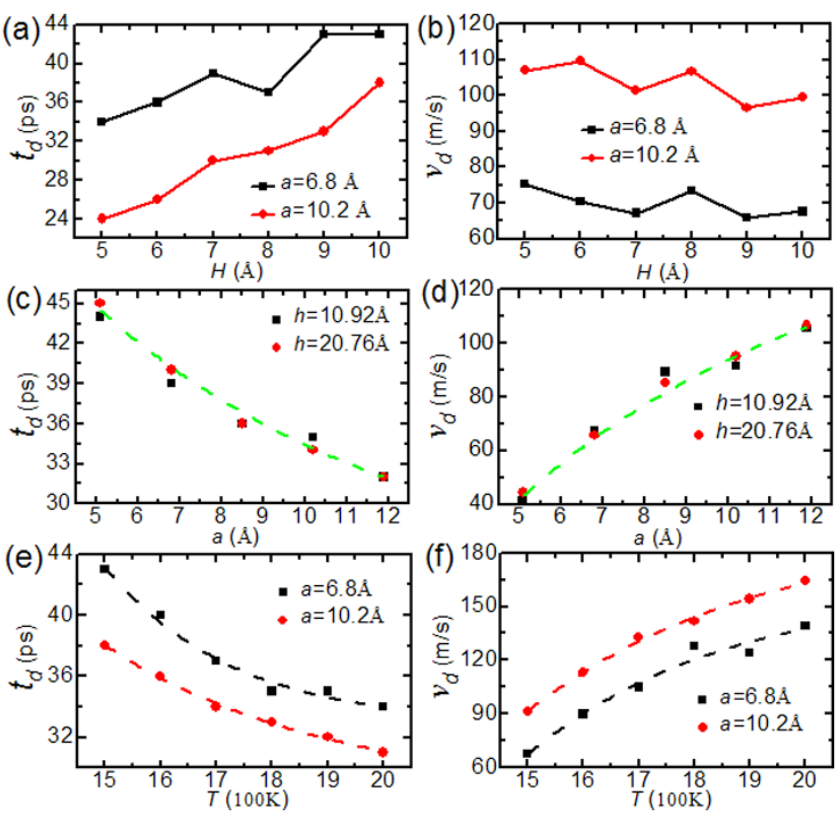

Figure $8 \mid$ Detaching properties of disk Cu on the PG2 surface at $1500 \mathrm{~K}$. Similarly, the $t_{d}\left(v_{d}\right)$ shows a increasing (decreasing) tendency with the increasing $H$, and displays exponential relations with $a$ and $T$.

Figure 8 presents the detaching properties of the $\mathrm{Cu}$ liquid droplet on the PG2 surfaces. Like the detachment on the PG surface, the detachment on the PG2 surface displays strong dependence on both the films and the substrates. Figure $8 \mathrm{a}$ and $\mathrm{b}$ show that the $t_{d}$ significantly increases but the $v_{d}$ slightly decreases with the increasing $H$. Additionally, both the $t_{d}$ and $v_{d}$ display an exponential relation with $a$ and temperature, as shown in Figures $8 \mathrm{c}-\mathrm{f}$. One difference is that both the gaps of $t_{d}$ and $v_{d}$ between $a=6.8$ and $10.2 \AA$ are larger than that in the $\mathrm{Cu}-\mathrm{PG}$ system, indicating that the detachment is more sensitive to $a$ in the Cu-PG2 system.

Figure 9 shows dewetting properties of liquid films on the horizontally placed carbon nanotubes (HCNT). Like the PG and PG2 surfaces, the HCNT could also detach the droplet, as shown in

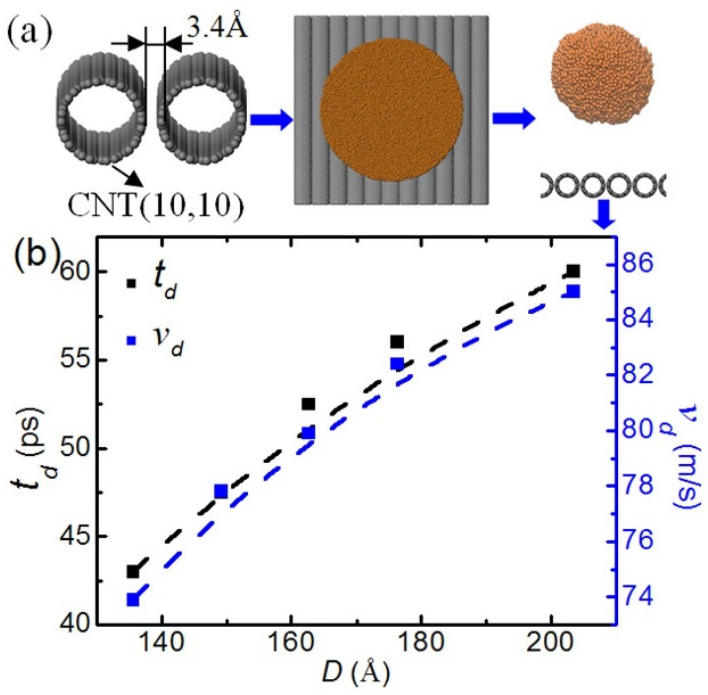

Figure 9 | Detaching properties of disk $\mathrm{Cu}$ on another C-based substrate. The $H$ is fixed at $10 \AA$. (a) Structure of the substrate that consists of horizontally placed carbon nanotubes (HCNTs) with fixed interval distance $3.4 \AA$, and partial snapshots of the detaching process. (b) Detaching properties of the disk with varying $D$ on the HCNT.
Figure 9a. Further research presents that both the $t_{d}$ and $v_{d}$ display an increasing tendency with increasing $D$, as shown in Figure $9 \mathrm{~b}$, which is consistent with the results in Figure 6, which reflects the importance of surface nanostructures on the special dewettability and indicates the effective method to control these properties by tuning the sizes of the $\mathrm{Cu}$ films.

\section{Discussion}

In summary, this biomimetic study on the wettability of nanopillared graphene reveals the importance of the surface nanostructures on the special dewettability. The MD results show that the CNT pillars act as the effective dewetting components, and this not only leads to the increase of dewettability but also brings great convenience in detaching metallic droplets from the surfaces. The inertia plays a dominant role in the dewetting dynamics, especially at high temperature. The detachment can be controlled by simply tunning the geometrical parameters of the films and the surface nanostructures, as well as the temperature. The switching dewetting mode and the controlled detachment not only shed new light on the special dewttability of nanopillared surfaces, but also pave the way for new and promising applications of these surfaces in daily life, self-cleaning materials, nanofluidic devices and homogeneous coatings.

\section{Methods}

The Cu nanodisk is extracted from a bulk liquid, then deposited at a distance of $3.0 \AA$ above the PG surface. The CNT pillars are placed on the $\mathrm{G}$ to mimic the lotus leaves which possess superhydrophobicity and self-cleaning effects ${ }^{4,5}$. MD simulations are performed to study the dewetting properties of metallic liquid films on PG under the constant-volume and constant-temperature (NVT) ensemble, by using the largescale atomic/molecular massively parallel simulator (LAMMPS) package $\mathrm{e}^{43,44}$. The interaction among metal atoms is described by an embedded atom method (EAM) potential ${ }^{45}$. The $\mathrm{C}-\mathrm{C}$ interaction is modeled by an adaptive intermolecular reactive empirical bond order (AIREBO) potential ${ }^{46}$. due to the fact that metal and carbon can only form soft bonds via charge transfer from the $\pi$ electrons in the $s p^{2}$ hybridized carbon to the empty $4 \mathrm{~s}$ states of metal ${ }^{47,48}$, we utilize the 12-6 Lennard-Jones (LJ) potential with a well depth $\varepsilon=0.018 \mathrm{eV}$ and size parameter $\sigma=3.0 \AA$ to describe the metal-C interaction ${ }^{38,49}$. This LJ potential was found to reproduce the equilibrium CA as $132^{\circ}$ for $\mathrm{Cu}$ on pristine graphene. These values are in good agreement with the experimental one $140^{\circ 50}$. The temperature is controlled by the Nose-Hoover method and the time integration of the Newton's equation of motion is undertaken using the velocity Verlet algorithm ${ }^{51}$ with a time step of $1.0 \mathrm{fs}$. All of the substrates are fixed during the MD simulations.

The CA of a liquid metal droplet on surfaces is calculated by the method used by Werder ${ }^{52}$. Firstly, the isochore line of the time-averaged metal number density being about one fourth of the bulk density is defined as liquid-air interface. Secondly, we fit a circle to the interface, and then measure the CA. The velocity of the liquid nanodroplet along the $z$ direction vertical to the substrate is defined as $v_{\mathrm{z}}(t)=\frac{d Z_{C M}}{d t}$, where $Z_{C M}$ is the displacement of the nanodroplet center of mass, and $t$ is time. The moment when the C-metal potential energy $E_{\mathrm{C} \text {-metal }}$ equals zero is defined as the detaching time $t_{d}$. After detachment, the liquid droplets remain at a constant velocity, which is defined as the detaching velocity $v_{d}$.

1. Autumn, K. et al. Adhesive force of a single gecko foot-hair. Nature 405, 681-685 (2000).

2. Geim, A. K. et al. Microfabricated adhesive mimicking gecko foot-hair. Nature Mater. 1, 1-3 (2003).

3. Lee, H., Lee, B. P. \& Messersmith, P. B. A reversible wet/dry adhesive inspired by mussels and geckos. Nature 448, 338-341 (2007).

4. Barthlott, W. \& Neinhuis, C. Purity of the sacred lotus, or escape from contamination in biological surfaces. Planta 202, 1-8 (1997).

5. Neinhuis, C. \& Barthlott, W. Characterization and distribution of water-repellent, self-cleaning plant surfaces. Ann. Bot. 79, 667-677 (1997).

6. Yuan, Q. \& Zhao, Y.-P. Wetting on flexible hydrophilic pillar-arrays. Sci. Rep. 3, 1944; DOI:10.1038/srep01944 (2013).

7. Feng, X. J. \& Jiang, L. Design and creation of uperwetting/antiwetting surfaces. Adv. Mater. 18, 3063-3078 (2006).

8. Minko, S. et al. Two-level structured self-adaptive surfaces with reversibly tunable properties. J. Am. Chem. Soc. 125, 3896-3900 (2003).

9. Dong, J., Yao, Z. H., Yang, T. Z., Jiang, L. L. \& Shen, C. M. Control of Superhydrophilic and Superhydrophobic Graphene Interface. Sci. Rep. 3, 1733; DOI:10.1038/srep01733 (2013).

10. Meng, S., Zhang, Z. Y. \& Kaxiras, E. Tuning solid surfaces from hydrophobic to superhydrophilic by submonolayer surface modification. Phys. Rev. Lett. 97, 036107 (2006). 
11. Xie, X. et al. Large-range Control of the Microstructures and Properties of Threedimensional Porous Graphene. Sci. Rep. 3, 2117; DOI:10.1038/srep02117 (2013).

12. Zhang, L., Zhong, Y., Cha, D. \& Wang, P. A self-cleaning underwater superoleophobic mesh for oil-water separation. Sci. Rep. 3, 2326; DOI:10.1038/ srep02326 (2013).

13. Chen, P. \& Xu, Z. Mineral-Coated Polymer Membranes with Superhydrophilicity and Underwater Superoleophobicity for Effective Oil/Water Separation. Sci. Rep. 3, 2776; DOI:10.1038/srep02776 (2013)

14. Herminghaus, S., Lenz, P. \& Lipowsky, R. Liquid morphologies on structured surfaces: from microchannels to microchips. Science 283, 46-49 (1999).

15. Feng, L. et al. Super-hydrophobic surface of aligned polyacrylonitrile nanofibers. Angew. Chem. Int. Ed. 41, 1221-1223 (2002).

16. Sun, T. L., Feng, L., Gao, X. F. \& Jiang, L. Bioinspired surfaces with special wettability. Acc. Chem. Res. 38, 644-652 (2005)

17. Wang, R. et al. Light-induced amphiphilic surfaces. Nature 388, 431-432 (1997).

18. Feng, L. et al. Super-hydrophobic surfaces: from natural to artificial. Adv. Mater. 14, 1857-1860 (2002).

19. Blossey, R. Self-cleaning surfaces virtual realities. Nature Mater. 2, 301-306 (2003).

20. Lafuma, A. \& Quéré, D. Superhydrophobic states. Nature Mater. 2, 457-460 (2003).

21. Luna, M., Colchero, J. \& Baró, A. M. Study of water droplets and films on graphite by noncontact scanning force microscopy. J. Phys. Chem. B 103, 9576-9581 (1999).

22. Koishi, T., Yasuoka, K., Fujikawa, S. \& Zeng, X. C. Measurement of contact-angle hysteresis for droplets on nanopillared surface and in the Cassie and Wenzel states: a molecular dynamics simulation study. ACS Nano 5, 6834-6842 (2011).

23. Zhu, C. Q., Li, H., Huang, Y. F., Zeng, X. C. \& Meng, S. Microscopic insight into surface wetting: relations between interfacial water structure and the underlying lattice constant. Phys. Rev. Lett. 110, 126101 (2013).

24. Damman, P., Baudelet, N. \& Reiter, G. Dewetting near the glass transition transition from a capillary force dominated to a dissipation dominated regime. Phys. Rev. Lett. 91, 216101 (2003).

25. Yerushalmi-Rozen, R., Klein, J. \& Fetters, L. J. Suppression of rupture in thin, nonwetting liquid films. Science. 263, 793-795 (1994).

26. Reiter, G. Auto-optimization of dewetting rates by rim instabilities in slipping polymer films. Phys. Rev. Lett. 91, 166103 (2003).

27. Huang, W. Y., Qian, W. \& El-Sayed, M. A. Gold nanoparticles propulsion from surface fueled by absorption of femtosecond laser pulse at their surface plasmon resonance. J. Am. Chem. Soc. 128, 13330-13331 (2006).

28. Trice, J., Thomas, D., Favazza, C., Sureshkumar, R. \& Kalyanaraman, R. Pulsedlaser-induced dewetting in nanoscopic metal films: theory and experiments. Phys. Rev. B 75, 235439 (2007)

29. Mukherjee, R. et al. Stability and wewetting of metal nanoparticle filled thin polymer films: control of instability length scale and dynamics. ACS Nano 4 3709-3724 (2010).

30. Bischof, J., Scherer, D., Herminghaus, S. \& Leiderer, P. Dewetting modes of thin metallic films: nucleation of holes and spinodal dewetting. Phys. Rev. Lett. 77, 1536-1539 (1996).

31. Herminghaus, S. et al. Spinodal dewetting in liquid crystal and liquid metal films. Science 282, 916-919 (1998).

32. Chang, C. S., Kostylev, M. \& Ivanov, E. Metallic spintronic thin film as a hydrogen sensor. Appl. Phys. Lett. 102, 142405 (2013).

33. Hecht, D. S., Hu, L. B. \& Irvin, G. Emerging transparent electrodes based on thin films of carbon nanotubes, graphene, and metallic nanostructures. Adv. Mater. 23, 1482-1513 (2011).

34. Bozano, L. D. et al. Organic materials and thin-film structures for cross-point memory. Cells based on trapping in metallic nanoparticles. Adv. Funct. Mater. 15 1933-1939 (2005)

35. Favazza, C., Kalyanaraman, R. \& Sureshkumar, R. Robust nanopatterning by laser-induced dewetting of metal nanofilms. Nanotechnology 17, 4229-4234 (2006).

36. Rack, P. D., Guan, Y. F., Fowlkes, J. D., Melechko, A. V. \& Simpson, M. L. Pulsed laser dewetting of patterned thin metal films: A means of directed assembly. Appl. Phys. Lett. 92, 223108 (2008).

37. Habenicht, A., Olapinski, M., Burmeister, F., Leiderer, P. \& Boneberg, J. Jumping nanodroplets. Science 309, 2043-2045 (2005).
38. Afkhami, S. \& Kondic, L. Numerical simulation of ejected molten metal nanoparticles liquified by laser irradiation: Interplay of geometry and dewetting. Phys. Rev. Lett. 111, 034501 (2013).

39. Fuentes-Cabrera, M. et al. Controlling the velocity of jumping nanodroplets via their initial shape and temperature. ACS Nano 5, 7130-7136 (2011).

40. Hernández, S. C. et al. Chemical gradients on graphene to drive droplet motion. ACS Nano 7, 4746-4755 (2013).

41. Fitz-Gerald, J. M., Piqué, A., Chrisey, D. B., Rack, P. D. \& Zeleznik, M. Laser direct writing of phosphor screens for high-definition displays. Appl. Phys. Lett. 76, 1386-1388 (2000).

42. Wu, Y. Y., Fowlkes, J. D., Rack, P. D., Diez, J. A. \& Kondic, L. On the breakup of patterned nanoscale copper rings into droplets via pulsed-laser-induced dewetting: Competing liquid-phase instability and transport mechanisms. Langmuir 26, 11972-11979 (2011).

43. Fowlkes, J. D., Kondic, L., Diez, J., Wu, Y. Y. \& Rack, P. D. Self-assembly versus directed assembly of nanoparticles via pulsed laser induced dewetting of patterned metal films. Nano Lett. 11, 2478-2485 (2011).

44. Plimpton, S. Fast parallel algorithms for short-range molecular dnamics. J. Comput. Phys. 117, 1-19 (1995).

45. Zhao, H., Min, K. \& Aluru, N. R. Size and chirality dependent elastic properties of graphene nanoribbons under uniaxial tension. Nano Lett. 9, 3012-3015 (2009).

46. Zhou, X. W. et al. Atomic scale structure of sputtered metal multilayers. Acta Mater. 49, 4005-15 (2001).

47. Stuart, S. J., Tutein, A. B. \& Harrison, J. A. A Reactive potential for hydrocarbons with intermolecular interactions. J. Chem. Phys. 112, 6472-6486 (2000).

48. Sutter, P., Hybertsen, M. S., Sadowski, J. T. \& Sutter, E. Electronic structure of fewlayer epitaxial graphene on $\mathrm{Ru}(0001)$. Nano Lett. 9, 2654-2660 (2009).

49. Deng, B., Xu, A. W., Chen, G. Y., Song, R. Q. \& Chen, L. P. Synthesis of coppercore/carbon-sheath nanocables by a surfactant-assisted. Hydrothermal reduction/carbonization process. J. Phys. Chem. B 110, 11711-11716 (2006).

50. Fuentes-Cabrera, M. et al. Molecular dynamics study of the dewetting of copper on graphite and graphene: Implications for nanoscale self-assembly. Phys. Rev. E 83, 041603 (2011).

51. Naidich, Y. V. \& Kolesnichenko, G. A. A study of wetting of diamond and graphite by fused metals and alloys VII. The effect of vanadium, niobium, manganese, molybdenum, and tungsten on wetting of graphite by copper-based alloys. Powder Metall. Met. Ceram. 7, 563-565 (1968).

52. Werder, T., Walther, J. H., Jaffe, R. L., Halicioglu, T. \& Koumoutsakos, P. On the Water-Carbon Interaction for Use in Molecular dynamics simulations of graphite and carbon nanotubes. J. Phys. Chem. B 107, 1345-1352 (2003).

\section{Acknowledgments}

The authors would like to acknowledge the support from the National Natural Science Foundation of China (Grant No. 51271100). This work is also supported by the National Basic Research Program of China (Grant No.2012CB825702). This work is also supported by the Special Funding in the Project of the Taishan Scholar Construction Engineering.

\section{Author contributions}

X.Y.L. designed the specific mathematical analysis, performed all simulations and theoretical approximations, and prepared the manuscript. X.Y.L. and H.L. discussed the results, drew conclusions and edited the manuscript. H.L., Y.Z.H., Y.W. and J.C.D. supervised the study.

\section{Additional information}

Supplementary information accompanies this paper at http://www.nature.com/ scientificreports

Competing financial interests: The authors declare no competing financial interests

How to cite this article: Li, X.Y., He, Y.Z., Wang, Y., Dong, J.C. \& Li, H. Dewetting Properties of Metallic Liquid Film on Nanopillared Graphene. Sci. Rep. 4, 3938; DOI:10.1038/srep03938 (2014)

This work is licensed under a Creative Commons AttributionNonCommercial-NoDerivs 3.0 Unported license. To view a copy of this license, visit http://creativecommons.org/licenses/by-nc-nd/3.0 